\title{
Stable Dynamics of Sand Automata
}

\author{
Alberto Dennunzio ${ }^{1}$, Pierre Guillon ${ }^{2}$, and Benoît Masson ${ }^{3}$ \\ 1 Università degli studi di Milano-Bicocca, Dipartimento di Informatica Sistemistica e \\ Comunicazione, viale Sarca 336, 20126 Milano, Italy \\ dennunzio@disco.unimib.it \\ 2 Université Paris-Est, Laboratoire d'Informatique de l'Institut Gaspard Monge, UMR \\ CNRS 8049, 5 bd Descartes, 77454 Marne la Vallée Cedex 2, France \\ pierre.guillon@univ-mlv.fr \\ 3 Laboratoire d'Informatique Fondamentale de Marseille (LIF)-CNRS, Aix-Marseille \\ Université, 39 rue Joliot-Curie, 13453 Marseille Cedex 13, France \\ benoit.masson@lif .univ-mrs.fr
}

\begin{abstract}
In this paper, we study different notions of stability for sand automata, dynamical systems inspired by sandpile models and cellular automata. First, we study the topological stability properties of equicontinuity and ultimate periodicity, proving that they are equivalent. Then, we deal with nilpotency. The classical definition for cellular automata being meaningless in that setting, we define a more suitable one. Finally, we prove that this dynamical behavior is undecidable.
\end{abstract}

\section{Introduction}

Self-organized criticality (SOC [2]) is a common phenomenon observed in a huge variety of processes in physics, biology and computer science. A SOC system evolves to a "critical state" after some finite transient. Examples of SOC systems are: sandpiles, snow avalanches, star clusters in the outer space, earthquakes, forest fires, load balance in operating systems [1]. Among them, sandpile models are a paradigmatic formal model for SOC systems [10].

In [3], the authors introduced sand automata as a generalization of sandpile models and transposed them in the setting of discrete dynamical systems. A key-point of [3] was to introduce a (locally compact) metric topology to study the dynamical behavior of sand automata. A first and important result was a fundamental representation theorem similar to the well-known theorem of Hedlund for cellular automata [11, 3]. In [4, 5], the authors investigate sand automata by dealing with some basic set properties and decidability issues. Then, in [8], a new compact topology is introduced, inspired by the strong relation between sand automata and cellular automata. It is proved that with

\footnotetext{
* This work has been supported by the Interlink/MIUR project "Cellular Automata: Topological Properties, Chaos and Associated Formal Languages", by the ANR Blanc "Projet Sycomore" and by the PRIN/MIUR project "Formal Languages and Automata: Mathematical and Applicative Aspects".
}

Please use the following format when citing this chapter:

Dennunzio, A., Guillon, P. and Masson, B., 2008, in IFIP International Federation for Information Processing, Volume 273; Fifth IFIP International Conference on Theoretical Computer Science; Giorgio Ausiello, Juhani Karhumäki, Giancarlo Mauri, Luke Ong; (Boston: Springer), pp. $157-169$. 
this new topology, the representation theorem still holds, while the compactness provides new opportunities for further topological studies of the model.

In this paper we continue the study of sand automata dynamics of [4,5], using the topological framework from [8]. We focus on stability, which is a major issue for isolating the realistic sandpile models satisfying the SOC principles. More precisely, we study different types of stability. First, we deal with the topological stability, i.e., the equicontinuity and ultimate periodicity properties. We prove that they are equivalent. We also show the insignificance of expansivity, a form of strong instability. This fact suggests that the topological classification for cellular automata from [13] cannot be easily generalized to sand automata.

Then, we study nilpotency, a very strong form of dynamical stability. The classical definition of nilpotency for cellular automata [7, 12] is no more meaningful here, since it would prevent any sand automaton from being nilpotent. Therefore, we introduce a new definition which captures the intuitive idea that a nilpotent automaton destroys all configurations: a sand automaton is nilpotent if all configurations get closer and closer to a uniform configuration, not necessarily reaching it. Finally, we prove that this behavior is undecidable, using the undecidability of the nilpotency of spreading cellular automata.

The paper is structured as follows. Section 2 recalls basic definitions and results on cellular automata and sand automata. In Section 3, results on the topological stability of sand automata are proved and discussed. Nilpotency of sand automata is then defined and proved undecidable in Section 4.

\section{Definitions}

For all $a, b \in \mathbb{Z}$ with $a \leq b$, let $[a, b]=\{a, a+1, \ldots, b\}$ and $\widetilde{[a, b}]=[a, b] \cup$ $\{+\infty,-\infty\}$. Let $\mathbb{N}_{+}$be the set of positive integers.

For a vector $i \in \mathbb{Z}^{d}$, denote by $|i|$ the infinite norm of $i$. Let $A$ a (possibly infinite) alphabet, and $r \in \mathbb{N}, d \in \mathbb{N}_{+}$. Denote by $\mathcal{M}_{r}^{d}$ the set of all the $d$ dimensional matrices with values in $A$ and entry vectors in the hyper-rectangle $[-r, r]^{d}$.

\subsection{Cellular Automata}

Let $A$ be a finite alphabet. A $C A$ configuration of dimension $d$ is a function from $\mathbb{Z}^{d}$ to $A$. The set $A^{\mathbb{Z}^{d}}$ of all the CA configurations is called the $C A$ configuration space. This space is usually equipped with the Tychonoff metric $\mathrm{d}_{T}$ defined by

$$
\forall x, y \in A^{\mathbb{Z}^{d}}, \quad \mathrm{~d}_{T}(x, y)=2^{-k} \quad \text { where } \quad k=\min \left\{|j|: j \in \mathbb{Z}^{d}, x_{j} \neq y_{j}\right\} .
$$


The topology induced by $\mathrm{d}_{T}$ coincides with the product topology induced by the discrete topology on $A$. It makes the CA configuration space is a Cantor space: it is compact, perfect (i.e., it has no isolated points) and totally disconnected.

A cellular automaton (CA) is a quadruple $\langle A, d, r, g\rangle$, where $A$ is the alphabet, also called the state set, $d \in \mathbb{N}_{+}$is the dimension, $r \in \mathbb{N}$ is the radius and $g: \mathcal{M}_{r}^{d} \rightarrow A$ is the local rule of the automaton. The local rule $g$ induces a global rule $G: A^{\mathbb{Z}^{d}} \rightarrow A^{\mathbb{Z}^{d}}$ defined as follows,

$$
\forall x \in A^{\mathbb{Z}^{d}}, \forall i \in \mathbb{Z}^{d}, \quad G(x)_{i}=g\left(M_{r}^{i}(x)\right),
$$

where $M_{r}^{i}(x) \in \mathcal{M}_{r}^{d}$ is the finite portion of $x$ of reference position $i \in \mathbb{Z}^{d}$ and radius $r$ defined by $\forall k \in[-r, r]^{d}, M_{r}^{i}(x)_{k}=x_{i+k}$.

For any $k \in \mathbb{Z}^{d}$ the shift map $\sigma^{k}: A^{\mathbb{Z}^{d}} \rightarrow A^{\mathbb{Z}^{d}}$ is defined by $\forall x \in A^{\mathbb{Z}^{d}}, \forall i \in \mathbb{Z}^{d}$, $\sigma^{k}(x)_{i}=x_{i+k}$. A function $F: A^{\mathbb{Z}^{d}} \rightarrow A^{\mathbb{Z}^{d}}$ is said to be shift-commuting if $\forall k \in$ $\mathbb{Z}^{d}, F \circ \sigma^{k}=\sigma^{k} \circ F$. Note that CA are exactly the class of all shift-commuting functions which are (uniformly) continuous with respect to the Tychonoff metric (Hedlund's theorem from [11]). For the sake of simplicity, we will make no distinction between a CA and its global rule $G$.

For a given CA, a state $s \in A$ is quiescent (resp., spreading) if for all matrices $U \in \mathcal{M}_{r}^{d}$ such that $\forall k \in[-r, r]^{d}$, (resp., $\exists k \in[-r, r]^{d}$ ) $U_{k}=s$, it holds that $g(U)=s$. Remark that a spreading state is also quiescent. A CA is said to be spreading if it has a spreading state. In the sequel, the spreading state of any spreading $\mathrm{CA}$ will be denoted $0 \in A$.

\subsection{SA Configurations}

A $S A$ configuration (or simply configuration) is a set of sand grains organized in piles and distributed all over the $d$-dimensional lattice $\mathbb{Z}^{d}$. A pile is an element of $\widetilde{\mathbb{Z}}=\mathbb{Z} \cup\{-\infty,+\infty\}$ which represents a number of grains. One pile is positioned in each point of the lattice $\mathbb{Z}^{d}$. Formally, a configuration $x$ is a function from $\mathbb{Z}^{d}$ to $\widetilde{\mathbb{Z}}$ which associates any vector $i=\left(i_{1}, \ldots, i_{d}\right) \in \mathbb{Z}^{d}$ with the number $x_{i} \in \widetilde{\mathbb{Z}}$ of grains in the pile of position $i$. When the dimension $d$ id known without ambiguity, we note 0 the null vector of $Z^{d}$. Denote by $\mathcal{C}=\widetilde{\mathbb{Z}}^{\mathbb{Z}^{d}}$ the set of all configurations.

A configuration $x \in \mathcal{C}$ is said to be constant if there is an integer $c \in \mathbb{Z}$ such that for any vector $i \in \mathbb{Z}^{d}, x_{i}=c$. In that case we write $x=\underline{c}$. A configuration $x \in \mathcal{C}$ is said to be bounded if there exist two integers $m_{1}, m_{2} \in \mathbb{Z}$ such that for all vectors $i \in \mathbb{Z}^{d}, m_{1} \leq x_{i} \leq m_{2}$. Denote by $\mathcal{B}$ the set of all bounded configurations.

A measuring device $\beta_{r}^{m}$ of precision $r \in \mathbb{N}$ and reference height $m \in \mathbb{Z}$ is a function from $\widetilde{\mathbb{Z}}$ to $\widetilde{[-r, r]}$ defined as follows 


$$
\forall n \in \widetilde{\mathbb{Z}}, \quad \beta_{r}^{m}(n)= \begin{cases}+\infty & \text { if } n>m+r \\ -\infty & \text { if } n<m-r \\ n-m & \text { otherwise. }\end{cases}
$$

A measuring device is used to evaluate the relative height of two piles, with a bounded precision. This is the technical basis of the definition of cylinders, distance and ranges which are used all along this article.

In [8], a topology, inspired by the topology on CA configurations, is defined as follows.

Definition 1 (cylinder). For any configuration $x \in \mathcal{C}$, for any $r \in \mathbb{N}$, and for any $i \in \mathbb{Z}^{d}$, the cylinder of $x$ centered on $i$ and of radius $r$ is the $d$-dimensional matrix $C_{r}^{i}(x) \in \mathcal{M}_{r}^{d}$ defined on the finite alphabet $\widetilde{[-r, r]}$ by

$$
\forall k \in[-r, r]^{d}, \quad\left(C_{r}^{i}(x)\right)_{k}=\beta_{r}^{0}\left(x_{i+k}\right) .
$$

Definition 2. For any pair of configurations $x, y \in \mathcal{C}$, we define

$$
\mathrm{d}(x, y)=2^{-k} \quad \text { where } \quad k=\min \left\{r \in \mathbb{N}: C_{r}^{0}(x) \neq C_{r}^{0}(y)\right\} .
$$

As a consequence, two configurations $x, y$ are compared by putting boxes (the cylinders) at height 0 around the corresponding piles indexed by 0 . The integer $k$ is the size of the smallest cylinders in which a difference appears between $x$ and $y$.

With the topology induced by $\mathrm{d}$, the SA configuration space is perfect, totally disconnected, and, unlike the original topology used in [11, 3], compact (see [8]).

\subsection{Sand Automata}

For any integer $r \in \mathbb{N}$, for any configuration $x \in \mathcal{C}$ and any index $i \in \mathbb{Z}^{d}$ with $x_{i} \neq \pm \infty$, the range of center $i$ and radius $r$ is the $d$-dimensional matrix $R_{r}^{i}(x) \in \mathcal{M}_{r}^{d}$ on the finite alphabet $A=\widehat{[-r, r]} \cup \perp$ such that

$$
\forall k \in[-r, r]^{d}, \quad\left(R_{r}^{i}(x)\right)_{k}= \begin{cases}\perp & \text { if } k=0, \\ \beta_{r}^{x_{i}}\left(x_{i+k}\right) & \text { otherwise. }\end{cases}
$$

The range is used to define a sand automaton. It is a kind of cylinder, where the observer is always located on the top of the pile $x_{i}$ (called the reference). It represents what the automaton is able to see at position $i$. Sometimes the central $\perp$ symbol may be omitted for simplicity sake. The set of all possible ranges of radius $r$, in dimension $d$, is denoted by $\mathcal{R}_{r}^{d}$.

A sand automaton ( $\mathrm{SA}$ ) is a deterministic finite automaton working on configurations. Each pile is updated synchronously, according to a local rule which computes the variation of the pile by means of the range. Formally, a SA is a 
triple $\langle d, r, f\rangle$, where $d$ is the dimension, $r$ is the radius and $f: \mathcal{R}_{r}^{d} \rightarrow[-r, r]$ is the local rule of the automaton. The global rule $F: \mathcal{C} \rightarrow \mathcal{C}$ is defined by

$$
\forall x \in \mathcal{C}, \forall i \in \mathbb{Z}^{d}, \quad F(x)_{i}= \begin{cases}x_{i} & \text { if } x_{i}= \pm \infty \\ x_{i}+f\left(R_{r}^{i}(x)\right) & \text { otherwise. }\end{cases}
$$

The following example illustrates a sand automaton whose behavior will be studied in Section 4. For more examples, we refer to [5].

Example 1 (the automaton $\mathcal{N}$ ). This automaton destroys a configuration by collapsing all piles towards the lowest one. It decreases a pile when there is a lower pile in the neighborhood. Let $\mathcal{N}=\left\langle 1,1, f_{\mathcal{N}}\right\rangle$ of global rule $F_{\mathcal{N}}$ where

$$
\forall a, b \in \widetilde{[-1,1]}, \quad f_{\mathcal{N}}(a, b)=\left\{\begin{aligned}
-1 & \text { if } a<0 \text { or } b<0 \\
0 & \text { otherwise. }
\end{aligned}\right.
$$

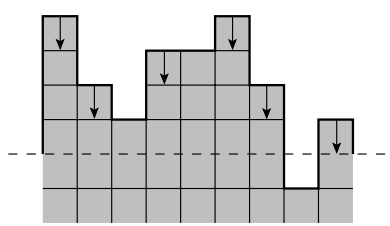

Fig. 1 Illustration of the behavior of $\mathcal{N}$.

When no misunderstanding is possible, we identify a SA with its global rule $F$. For any $k \in \mathbb{Z}^{d}$, we extend the definition of the shift map to $\mathcal{C}, \sigma^{k}: \mathcal{C} \rightarrow \mathcal{C}$ is defined by $\forall x \in \mathcal{C}, \forall i \in \mathbb{Z}^{d}, \sigma^{k}(x)_{i}=x_{i+k}$. The raising map $\rho: \mathcal{C} \rightarrow \mathcal{C}$ is defined by $\forall x \in \mathcal{C}, \forall i \in \mathbb{Z}^{d}, \rho(x)_{i}=x_{i}+1$. A function $F: \mathcal{C} \rightarrow \mathcal{C}$ is said to be vertical-commuting if $F \circ \rho=\rho \circ F$. A function $F: \mathcal{C} \rightarrow \mathcal{C}$ is infinity-preserving if for any configuration $x \in \mathcal{C}$ and any vector $i \in \mathbb{Z}^{d}, F(x)_{i}=+\infty$ if and only if $x_{i}=+\infty$ and $F(x)_{i}=-\infty$ if and only if $x_{i}=-\infty$.

With the topology from [8], the Hedlund-like representation theorem for SA from [3] remains valid.

Theorem 1 ([8]). A mapping $F: \mathcal{C} \rightarrow \mathcal{C}$ is a $S A$ if and only if $F$ is (uniformly) continuous, shift-commuting, vertical-commuting and infinity-preserving.

\section{Some Dynamical Behaviors}

SA are very interesting models, whose complexity lies somewhere between $d$ dimensional and $d+1$-dimensional $\mathrm{CA}$. Indeed, the latter can simulate $d$ dimensional SA, which can, in turn, simulate the former [5, 8]. We are interested 
in studying the SA complexity from the stability point of view. The concepts that first come to mind to formalize the notion of stability are inspired by the topological classifications given in [9, 13] for CA. In [13], one-dimensional CA are classified into four classes, from the most stable to the most unstable behavior: equicontinuous $\mathrm{CA}$, non-equicontinuous $\mathrm{CA}$ admitting an equicontinuity configuration, sensitive but not positively expansive CA, positively expansive $\mathrm{CA}$. Things are very different as soon as we get into dimension $d=2$, as noted in $[15,14]$. The question is now whether the complexity of the SA model is closer to that of the lower or the higher-dimensional CA. In this section we consider the above mentioned concepts in the SA settings, and we introduce the notion of ultimate periodicity, useful for the characterization of SOC systems. We prove that there exist no positively expansive SA and characterize equicontinuous $\mathrm{SA}$ as the ultimately periodic SA.

First, recall basic definitions. Let $(X, m)$ be a metric space and let $H: X \rightarrow$ $X$ be a continuous application. An element $x \in X$ is an equicontinuity point for $H$ if for any $\varepsilon>0$, there exists $\delta>0$ such that for all $y \in X, m(x, y)<\delta$ implies that $\forall n \in \mathbb{N}, m\left(H^{n}(x), H^{n}(y)\right)<\varepsilon$. The map $H$ is equicontinuous if for any $\varepsilon>0$, there exists $\delta>0$ such that for all $x, y \in X, m(x, y)<\delta$ implies that $\forall n \in \mathbb{N}, m\left(H^{n}(x), H^{n}(y)\right)<\varepsilon$. An element $x \in X$ is ultimately periodic for $H$ if there exist two integers $n \geq 0$ (the preperiod) and $p>0$ (the period) such that $H^{n+p}(x)=H^{n}(x)$. $H$ is ultimately periodic if there exist $n \geq 0$ and $p>0$ such that $H^{n+p}=H^{n}$. If $X$ is compact, $H$ is equicontinuous (resp. ultimately periodic) iff all elements of $X$ are equicontinuity points (resp. ultimately periodic). $H$ is sensitive (to the initial conditions) if there is a constant $\varepsilon>0$ such that for all points $x \in X$ and all $\delta>0$, there is a point $y \in X$ and an integer $n \in \mathbb{N}$ such that $m(x, y)<\delta$ but $m\left(F^{n}(x), F^{n}(y)\right)>\varepsilon$. $H$ is positively expansive if there is a constant $\varepsilon>0$ such that for all distinct points $x, y \in X$, there exists $n \in \mathbb{N}$ such that $m\left(H^{n}(x), H^{n}(y)\right)>\varepsilon$.

The classification from [13] is no more relevant in the SA context since the class of positively expansive $\mathrm{SA}$ is empty. This result can be related to the absence of positively expansive two-dimensional CA (see [15]), though the proof is much different.

Proposition 1. There are no positively expansive $S A$.

Proof. Let $F$ be a SA and for any $k \in \mathbb{N}$, let $\delta=2^{-k}$. Take two distinct configurations $x, y \in \mathcal{C}$ such that $\forall i \in[-k, k]^{d}, x_{i}=y_{i}=+\infty$. By infinitypreservingness, we get $\forall n \in \mathbb{N}, \forall i \in[-k, k]^{d}, F^{n}(x)_{i}=F^{n}(y)_{i}=+\infty$, hence $d\left(F^{n}(x), F^{n}(y)\right)<\delta$.

We now prove that two different notions of stability, such as equicontinuity and ultimate periodicity, are equivalent. We need the following lemma, which allows a better understanding of equicontinuity for SA.

Lemma 1. If $F$ is an equicontinuous $S A$, then the variation of a pile is bounded by the differences in an initial neighborhood, i.e., there exists an integer $l \in \mathbb{N}$ such that all configurations $x \in \mathcal{C}$ with $x_{0}=0$ satisfy 


$$
\forall n \in \mathbb{N}, \quad\left|F^{n}(x)_{0}\right| \leq \max _{\substack{|i| \leq l \\\left|x_{i}\right|<\infty}}\left\{\left|x_{i}\right|\right\} .
$$

Proof. If $F$ is equicontinuous, in particular, for $\varepsilon=2^{0}$, there exists $\delta=2^{-l}$ such that for all $x, y \in \mathcal{C}$, if $C_{l}^{0}(x)=C_{l}^{0}(y)$, then $\forall n \in \mathbb{N}, C_{0}^{0}\left(F^{n}(x)\right)=C_{0}^{0}\left(F^{n}(y)\right)$. First, consider a configuration $y$ which has infinite l-neighborhood, i.e., $\forall i \in$ $[-l, l]^{d}, y_{i} \notin[-l, l]$. Let $z$ defined by $z_{i}=+\infty$ if $y_{i} \geq 0$ and $z_{i}=-\infty$ if $y_{i}<0$, in such a way that $C_{l}^{0}(y)=C_{l}^{0}(z)$. Then $\forall n \in \mathbb{N}, C_{0}^{0}\left(F^{n}(y)\right)=C_{0}^{0}\left(F^{n}(z)\right)=$ $C_{0}^{0}(z)$, i.e., $F^{n}(y)_{0}<-l \Leftrightarrow y_{0}<-l$ and $F^{n}(y)_{0}>l \Leftrightarrow y_{0}>l$.

Now, let $x \in \mathcal{C}$ such that $x_{0}=0$ and $m=\max _{|i| \leq l,\left|x_{i}\right|<\infty}\left\{\left|x_{i}\right|\right\}$. Notice that $\rho^{l+m+1}(x)$ has infinite $l$-neighborhood, since $x_{i} \leq m$ or $x_{i}=+\infty$ for $|i| \leq l$. Hence, as seen before, $\forall n \in \mathbb{N}, F^{n}(x)_{0} \leq m$. A symmetrical reasoning on $\rho^{-l-m-1}(x)$ gives $\forall n \in \mathbb{N},\left|F^{n}(x)_{0}\right| \leq m$.

Proposition 2. A SA is equicontinuous if and only if it is ultimately periodic.

Proof. Let $F$ be a SA such that with $F^{n+p}=F^{n}$ for some $n \geq 0, p>0$. Since $F, F^{2}, \ldots, F^{n+p-1}$ are uniformly continuous maps, for any $\varepsilon>0$ there exists $\delta>0$ such that for all $x, y \in \mathcal{C}$ with $\mathrm{d}(x, y)<\delta$, it holds that $\forall q \in \mathbb{N}, q<n+p$, $\mathrm{d}\left(F^{q}(x), F^{q}(y)\right)<\varepsilon$. Since for any $t \in \mathbb{N}, F^{t}$ is equal to some $F^{q}$ with $q<n+p$, the map $F$ is equicontinuous.

Let $F$ be an equicontinuous SA and $l$, as in Lemma 1 , such that for all $x, y \in \mathcal{C}$, if $C_{l}^{0}(x)=C_{l}^{0}(y)$, then $\forall n \in \mathbb{N}, C_{0}^{0}\left(F^{n}(x)\right)=C_{0}^{0}\left(F^{n}(y)\right)$. Let $x \in \mathcal{C}$ such that $x_{0}$ is finite. Should we vertically shift it, we can assume $x_{0}=0$. Let $y \in \mathcal{C}$ defined by $y_{i}=\max \left\{\min \left\{x_{i}, l+1\right\},-l-1\right\}$ if $|i| \leq l$ and $y_{i}=+\infty$ otherwise, in such a way that $C_{l}^{0}(x)=C_{l}^{0}(y)$. By Lemma $1, \forall i \in[-l, l]^{d}, \forall n \in$ $\mathbb{N},\left|F^{n}(y)_{i}\right| \leq 2 l+2$. So we can find some preperiod $q_{y}$ and some period $p_{y}$ such that $\forall i \in[-l, l]^{d}, F^{p_{y}+q_{y}}(y)_{i}=F^{q_{y}}(y)_{i}$. Since the other piles are infinite, and then invariant, we get $F^{p_{y}+q_{y}}(y)=F^{q_{y}}(y)$. As a consequence, $C_{0}^{0}\left(F^{p_{y}+q_{y}}(x)\right)=$ $C_{0}^{0}\left(F^{q_{y}}(x)\right)$. Define $p$ (resp., $\left.q\right)$ as the least common multiple (resp., maximum) of all $p_{y}$ (resp., $q_{y}$ ) for $y \in \mathcal{C}$ such that $\left|y_{i}\right| \leq l+1$ if $|i| \leq l$ and $y_{i}=+\infty$ otherwise. Then, for any $x \in \mathcal{C}, C_{0}^{0}\left(F^{p+q}(x)\right)=C_{0}^{0}\left(F^{q}(x)\right)$; in particular for vertical and horizontal shifts of $x$, which gives $F^{p+q}(x)=F^{q}(x)$.

An important open question in the dynamical behavior of SA is the existence of non-sensitive SA without any equicontinuity configuration. An example for two-dimensional CA is given in [14], but the involved method can hardly be adapted for SA. However, we conjecture that such SA exist, which would lead to a classification of SA into four classes: equicontinuous, admitting an equicontinuity configuration (but not equicontinuous), non-sensitive without equicontinuity configurations, sensitive. 


\section{Nilpotency}

In this section we give a definition of nilpotency, the most stable dynamics of a dynamical system, adapted to SA. Then, we prove that this nilpotency behavior is undecidable (Theorem 3).

\subsection{Nilpotency of $C A$}

Here we recall the basic definitions and properties of nilpotent CA. Nilpotency is among the simplest dynamical behavior that an automaton may exhibit. Intuitively, a system is nilpotent if it destroys every piece of information in any initial configuration, reaching a common constant configuration after a while. For CA, this is formalized as follows.

Definition 3 (CA nilpotency $[7,12])$. A CA $G$ is nilpotent if

$$
\exists c \in A, \quad \exists N \in \mathbb{N} \quad \forall x \in A^{\mathbb{Z}^{d}}, \quad \forall n \geq N, \quad G^{n}(x)=\underline{c} .
$$

Remark that, because of the compactness of the CA configuration space, a $\mathrm{CA}$ is nilpotent if and only if it is nilpotent for all initial configurations (i.e., all configurations eventually reach the same configuration).

Spreading CA have the following stronger characterization.

Proposition 3 ([6]). A $C A G$, with spreading state 0 , is nilpotent if and only if for all configurations $x \in A^{\mathbb{Z}^{d}}, \lim _{n \rightarrow \infty} \mathrm{d}_{T}\left(G^{n}(x), \underline{0}\right)=0$.

This equivalence is very useful since the CA nilpotency has been proved undecidable in [12], even for the restricted class of spreading CA.

Theorem 2 ([12]). For a given state $s$, it is undecidable to know whether a cellular automaton with spreading state $s$ is nilpotent.

\subsection{Nilpotency of $S A$}

A direct adaptation of Definition 3 to $\mathrm{SA}$ is vain. Indeed, assume $F$ is a SA of radius $r$. For any $k \in \mathbb{Z}^{d}$, consider the configuration $x^{k} \in \mathcal{B}$ defined by $x_{0}^{k}=k$ and $x_{i}^{k}=0$ for any $i \in \mathbb{Z}^{d} \backslash\{0\}$. Since the pile of height $k$ may decrease at most by $r$ during one step of evolution of the SA, and the other piles may increase at most by $r, x^{k}$ requires at least $\lceil k / 2 r\rceil$ steps to reach a constant configuration. Thus, there exists no common integer $n$ such that all configurations $x^{k}$ reach a constant configuration in time $n$. This is a major difference with $\mathrm{CA}$, which 
is essentially due to the unbounded set of states and to the infinity-preserving property.

Thus, we propose to label as nilpotent the SA which make every pile approach a constant value, but not necessarily reaching it ultimately. This nilpotency notion, inspired by Proposition 3, is formalized as follows for a SA F:

$$
\exists c \in \mathbb{Z}, \quad \forall x \in \mathcal{C}, \quad \lim _{n \rightarrow \infty} \mathrm{d}\left(F^{n}(x), \underline{c}\right)=0
$$

Remark that $c$ shall not be taken in the full state set $\widetilde{\mathbb{Z}}$, because allowing infinite values for $c$ would not correspond to the intuitive idea that a nilpotent SA "destroys" a configuration (otherwise, the raising map would be nilpotent). Anyway, this definition is not satisfying because of the vertical commutativity: two configurations which differ by a vertical shift reach two different configurations, and then no nilpotent SA may exist. A possible way to work around this issue is to make the limit configuration depend on the initial one:

$$
\forall x \in \mathcal{C}, \quad \exists c \in \mathbb{Z}, \quad \lim _{n \rightarrow \infty} \mathrm{d}\left(F^{n}(x), \underline{c}\right)=0
$$

Again, since SA are infinity-preserving, an infinite pile cannot be destroyed (nor, for the same reason, can an infinite pile be built from a finite one). Therefore nilpotency has to involve the configurations of $\mathbb{Z}^{\mathbb{Z}^{d}}$, i.e., the ones without infinite piles. Moreover, every configuration $x \in \mathbb{Z}^{\mathbb{Z}^{d}}$ made of regular steps (i.e., in dimension 1 , for all $i \in \mathbb{Z}, x_{i}-x_{i-1}=x_{i+1}-x_{i}$ ) is invariant by the SA rule (possibly composing it with the vertical shift). So it cannot reach nor approach a constant configuration. Thus, the larger reasonable set on which nilpotency might be defined is the set of bounded configurations $\mathcal{B}$. This leads to the following formal definition of nilpotency for SA.

\section{Definition 4 (SA nilpotency).}

$$
\forall x \in \mathcal{B}, \quad \exists c \in \mathbb{Z}, \quad \lim _{n \rightarrow \infty} \mathrm{d}\left(F^{n}(x), \underline{c}\right)=0 .
$$

The following proposition shows that the class of nilpotent SA is nonempty. Remark that similar nilpotent SA can be constructed with any radius and in any dimension.

Proposition 4. The $S A \mathcal{N}$ from Example 1 is nilpotent.

Proof. Let $x \in \mathcal{B}$, let $i \in \mathbb{Z}$ such that for all $j \in \mathbb{Z}, x_{j} \geq x_{i}$. Clearly, after $x_{i+1}-x_{i}$ steps, $F_{\mathcal{N}}^{x_{i+1}-x_{i}}(x)_{i+1}=F_{\mathcal{N}}^{x_{i+1}-x_{i}}(x)_{i}=x_{i}$. By immediate induction, we obtain that for all $j \in \mathbb{Z}$ there exists $n_{j} \in \mathbb{N}$ such that $F_{\mathcal{N}}^{n_{j}}(x)_{j}=x_{i}$, hence $\lim _{n \rightarrow \infty} \mathrm{d}\left(F_{\mathcal{N}}^{n}(x), \underline{x_{i}}\right)=0$. 


\subsection{Undecidability}

The main result of this section is that SA nilpotency is undecidable (Theorem 3), by reducing to it the nilpotency of spreading CA. This emphasizes the fact that the dynamical behavior of SA is very difficult to predict. We think that this result might be used as the reference undecidable problem for further questions on SA.

\section{Problem Nil}

INSTANCE: a $\mathrm{SA} \mathcal{A}=\langle d, r, \lambda\rangle$;

QUESTION: is $\mathcal{A}$ nilpotent?

\section{Theorem 3. The problem Nil is undecidable.}

Proof. This is proved by reducing Nil to the nilpotency of spreading cellular automata. Remark that it is sufficient to show the result in dimension 1 . Let $\mathcal{S}$ be a spreading cellular automaton $\mathcal{S}=\langle A, 1, s, g\rangle$ of global rule $G$, with finite set of integer states $A \subset \mathbb{N}$ containing the spreading state 0 . We simulate $\mathcal{S}$ with the sand automaton $\mathcal{A}=\langle 1, r=\max (2 s, \max A), f\rangle$ of global rule $F$ using the following technique, also developed in [5]. Let $\xi: A^{\mathbb{Z}} \rightarrow \mathcal{B}$ be a function which inserts markers every two cells in the CA configuration to obtain a bounded SA configuration. These markers allow the local rule of the SA to know the absolute state of each pile and behave as the local rule of the CA. To simplify the proof, the markers are put at height 0 (see Figure 2):

$$
\forall y \in A^{\mathbb{Z}}, \forall i \in \mathbb{Z}, \quad \xi(y)_{i}= \begin{cases}0 \text { (marker) } & \text { if } i \text { is odd } \\ y_{i / 2} & \text { otherwise. }\end{cases}
$$

This can lead to an ambiguity when all the states in the neighborhood of size $4 s+1$ are at state 0 , as shown in the picture. But as in this special case the state 0 is quiescent for $g$, this is not a problem: the state 0 is preserved, and markers are preserved.

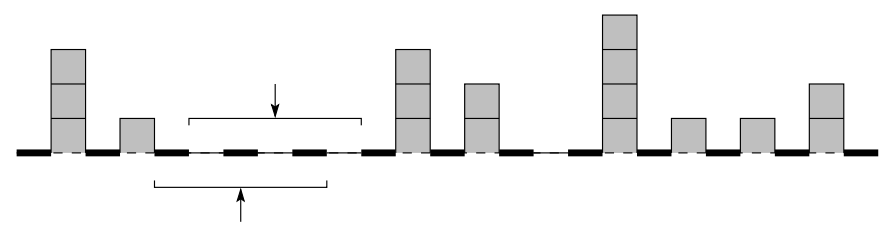

Fig. 2 Illustration of the function $\xi$ used in the simulation of the spreading CA $\mathcal{S}$ by $\mathcal{A}$. The thick segments are the markers used to distinguish the states of the CA, put at height 0 . There is an ambiguity for the two piles indicated by the arrows: with a radius 2 , the neighborhoods are the same, although one of the piles is a marker and the other the state 0 .

The local rule $f$ is defined as follows, for all ranges $R \in \mathcal{R}_{r}^{1}$, 


$$
f(R)=\left\{\begin{array}{r}
0 \quad \text { if } R_{-2 s+1}, R_{-2 s+3}, \ldots, R_{-1}, R_{1}, \ldots, R_{2 s-1} \in A \\
g\left(R_{-2 s}+a, R_{-2 s+2}+a, \ldots, R_{-2}+a, a, R_{2}+a, \ldots, R_{2 s}+a\right)-a \\
\text { if } R_{-2 s+1}=R_{-2 s+3}=\cdots=R_{2 s-1}=a<0 \text { and }-a \in A .
\end{array}\right.
$$

The first case is for the markers (and state 0) which remain unchanged, the second case is the simulation of $g$ in the even piles. As proved in [5], for any $y \in A^{\mathbb{Z}}$ it holds that $\xi(G(y))=F(\xi(y))$. The images by $f$ of the remaining ranges will be defined later on, first a few new notions need to be introduced.

A sequence of consecutive piles $\left(x_{i}, \ldots, x_{j}\right)$ from a configuration $x \in \mathcal{B}$ is said to be valid if it is part of an encoding of a CA configuration, i.e., $x_{i}=$ $x_{i+2}=\cdots=x_{j}$ (these piles are markers) and for all $k \in \mathbb{N}$ such that $0 \leq k<$ $(j-i) / 2, x_{i+2 k+1}-x_{i} \in A$ (this is a valid state). We extend this definition to configurations, when $i=-\infty$ and $j=+\infty$, i.e., $x \in \rho^{c} \circ \xi\left(A^{\mathbb{Z}}\right)$ for a given $c \in \mathbb{Z}$ ( $x \in \mathcal{B}$ is valid if it is the raised image of a $\mathrm{CA}$ configuration). A sequence (or a configuration) in invalid if it is not valid.

First we show that starting from a valid configuration, the $\mathrm{SA} \mathcal{A}$ is nilpotent if and only if $\mathcal{S}$ is nilpotent. This is due to the fact that we chose to put the markers at height 0 , hence for any valid encoding of the CA $x=\rho^{c} \circ \xi(y)$, with $y \in A^{\mathbb{Z}}$ and $c \in \mathbb{Z}$,

$$
\lim _{n \rightarrow \infty} \mathrm{d}_{T}\left(G^{n}(y), \underline{0}\right)=0 \quad \text { if and only if } \quad \lim _{n \rightarrow \infty} \mathrm{d}\left(F^{n}(x), \underline{c}\right)=0 .
$$

It remains to prove that for any invalid configuration, $\mathcal{A}$ is also nilpotent. In order to have this behavior, we add to the local rule $f$ the rules of the nilpotent automaton $\mathcal{N}$ for every invalid neighborhood of width $4 s+1$. For all ranges $R \in \mathcal{R}_{r}^{1}$ not considered in Equation (1),

$$
f(R)=\left\{\begin{aligned}
-1 & \text { if } R_{-r}<0 \text { or } R_{-r+1}<0 \text { or } \cdots \text { or } R_{r}<0 \\
0 & \text { otherwise. }
\end{aligned}\right.
$$

Let $x \in \mathcal{B}$ be an invalid configuration. Let $k \in \mathbb{Z}$ be any index such that $\forall l \in \mathbb{Z}, x_{l} \geq x_{k}$. Let $i, j \in \mathbb{Z}$ be respectively the lowest and greatest indices such that $i \leq k \leq j$ and $\left(x_{i}, \ldots, x_{j}\right)$ is valid ( $i$ may equal $j$ ). Remark that for all $n \in \mathbb{N},\left(F^{n}(x)_{i}, \ldots, F^{n}(x)_{j}\right)$ remains valid. Indeed, the markers are by construction the lowest piles and Equations (1) and (2) do not modify them. The piles coding for non-zero states can change their state by Equation (1), or decrease it by 1 by Equation (2), which in both cases is a valid encoding. Moreover, the piles $x_{i-1}$ and $x_{j+1}$ will reach a valid value after a finite number of steps: as long as they are invalid, they decrease by 1 until they reach a value which codes for a valid state. Hence, by induction, for any indices $a, b \in \mathbb{Z}$, there exists $N_{a, b}$ such that for all $n \geq N_{a, b}$ the sequence $\left(F^{n}(x)_{a}, \ldots, F^{n}(x)_{b}\right)$ is valid.

In particular, after $N_{-2 N r-1,2 N r+1}$ step, there is a valid sequence of length $4 N r+3$ centered on the origin (here, $N$ is the number of steps needed by $\mathcal{S}$ to reach the configuration $\underline{0}$, given by Definition 3). Hence, after $N_{-2 N r, 2 N r}+N$ 
steps, the local rule of the CA $\mathcal{S}$ applied on this valid sequence leads to 3 consecutive zeros at positions -1, 0, 1. All these steps are illustrated on Figure 3.

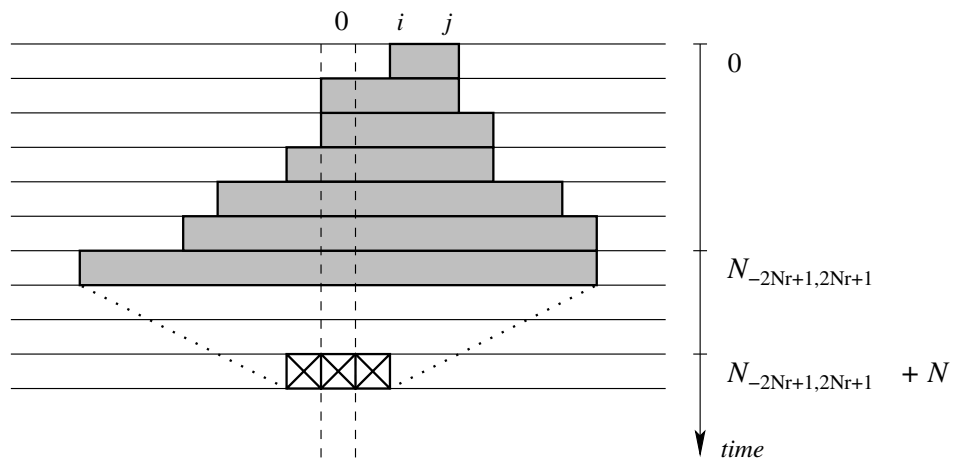

Fig. 3 Destruction of the invalid parts. The lowest valid sequence (in gray) extends until it is large enough. Then after $N$ other steps the 3 central piles (hatched) are destroyed because the rule of the CA is applied correctly.

In a similar way, we prove that for all $n \geq N_{-2 N r-k, 2 N r+k}+N$, the sequence $\left(F^{n}(x)_{-k}, \ldots, F^{n}(x)_{k}\right)$ is constant and does not evolve as $n$ grows. Therefore, there exists $c \in \mathbb{Z}$ such that $\lim _{n \rightarrow \infty} \mathrm{d}\left(F^{n}(x), \underline{c}\right)=0$. We just proved that $\mathcal{A}$ is nilpotent, i.e., $\lim _{n \rightarrow \infty} \mathrm{d}\left(F^{n}(x), \underline{c}\right)=0$ for all $x \in \mathcal{B}$, if and only if $\mathcal{S}$ is nilpotent (because of the equivalence of definitions given by Proposition 3 ), so Nil is undecidable (Theorem 2).

\section{Conclusion}

In this article we have continued the study of sand automata, using the compact topology on the SA configuration space introduced in [8]. This topology, inspired by the topology on CA, may facilitate studies about dynamical and topological properties of SA, as for the proof of the equivalence between equicontinuity and ultimate periodicity (Proposition 2).

Then, we have given a definition of nilpotency. Although it differs from the standard one for $\mathrm{CA}$, it captures the intuitive idea that a nilpotent automaton "destroys" configurations. Finally, we have proved that SA nilpotency is undecidable (Theorem 3). This fact enhances the idea that the behavior of a SA is hard to predict. We also think that this result might be used as a fundamental undecidability result, which could be reduced to other SA properties.

Besides, in the context of CA, nilpotency clearly implies ultimate periodicity. It appears that with our definitions, nilpotency of SA is not necessarily a 
particular case of ultimate periodicity $(\mathcal{N}$ is not ultimately periodic $)$. However, it would be interesting to see if it could be linked to other weaker stability notions.

Moreover, the study of global properties such as injectivity and surjectivity and their corresponding dimension-dependent decidability problems could help to understand if $d$-dimensional SA look more like $d$-dimensional or $d+1$ dimensional CA. Unfortunately, deciding these dynamical properties remains a major problem. Similarly, it would be interesting to solve the open question of the dichotomy between sensitive SA and those with equicontinuous configurations. A potential counter-example would give a more precise idea of the dynamical behaviors represented by SA.

\section{References}

1. P. Bak. How nature works - The science of SOC. Oxford University Press, 1997.

2. P. Bak, C. Tang, and K. Wiesenfeld. Self-organized criticality. Physical Review A, 38(1):364-374, 1988.

3. J. Cervelle and E. Formenti. On sand automata. In $20^{\text {th }}$ Symposium on Theoretical Aspects of Computer Science (STACS'03), volume 2607 of Lecture Notes in Computer Science, pages 642-653. Springer, 2003.

4. J. Cervelle, E. Formenti, and B. Masson. Basic properties for sand automata. In $30^{\text {th }}$ Conference on Mathematical Foundations of Computer Science (MFCS'O5), volume 3618 of Lecture Notes in Computer Science, pages 192-211. Springer, 2005.

5. J. Cervelle, E. Formenti, and B. Masson. From sandpiles to sand automata. Theoretical Computer Science, 381:1-28, 2007.

6. J. Cervelle and P. Guillon. Towards a Rice theorem on traces of cellular automata. In $32^{\text {nd }}$ Conference on Mathematical Foundations of Computer Science (MFCS'07), volume 4708 of Lecture Notes in Computer Science, pages 310-319. Springer, 2007.

7. K. Čulik, J. Pachl, and S. Yu. On the limit sets of cellular automata. SIAM Journal of Computing, 18(4):831-842, 1989.

8. A. Dennunzio, P. Guillon, and B. Masson. Topological properties of sand automata as cellular automata. In $1^{\text {st }}$ Symposium on Cellular Automata (JAC'08), 2008. To appear.

9. Robert H. Gilman. Classes of linear automata. Ergodic Theory \& Dynamical Systems, 7:105-118, 1988.

10. E. Goles and M. A. Kiwi. Game on line graphs and sandpile automata. Theoretical Computer Science, 115:321-349, 1993.

11. G. A. Hedlund. Endomorphisms and automorphisms of the shift dynamical system. Mathematical Systems Theory, 3:320-375, 1969.

12. J. Kari. The nilpotency problem of one-dimensional cellular automata. SIAM Journal of Computing, 21(3):571-586, 1992.

13. P. Kůrka. Languages, equicontinuity and attractors in cellular automata. Ergodic Theory ES Dynamical Systems, 17:417-433, 1997.

14. M. Sablik and G. Theyssier. Topological dynamics of $2 \mathrm{D}$ cellular automata. In $4^{\text {th }}$ Conference on Computability in Europe (CiE'08), volume 5028 of Lecture Notes in Computer Science. Springer, 2008. To appear.

15. M. A. Shereshevsky. Expansiveness, entropy and polynomial growth for groups acting on subshifts by automorphisms. Indagationes Mathematicae, 4(2):203-210, 1993. 